\title{
VALIDATION OF THE QUANTITATION METHODS OF 1-( $\beta$-PHENYLETHYL)-4-AMINO-1,2,4- TRIAZOLE BROMIDE SUBSTANCE BY SPECTROPHOTOMETRIC METHOD
}

\author{
KUCHERENKO LYUDMILA IVANOVNA ${ }^{1,2}$, PARNIUK NATALIA VIKTOROVNA ${ }^{1 *}$, KHROMYLOVA OLGA \\ VLADIMIROVNA ${ }^{1}$
}

${ }^{1}$ Department of Pharmaceutical Chemistry, Zaporizhzhia State Medical University, Zaporizhzhia, Ukraine. ${ }^{2}$ Scientific and Production Association "Farmatron”, Zaporizhzhia, Ukraine. Email: natalia.parniuk@gmail.com

Received: 13 July 2017, Revised and Accepted: 14 November 2017

\section{ABSTRACT}

Objective: The aim of the work was to determine the validation characteristics for the developed method of quantitation of 1 -( $\beta$-phenylethyl)-4amino-1,2,4-triazole bromide substance by absorption spectrophotometry in the ultraviolet region (UV).

Methods: For study purposes, the 1-( $\beta$-phenylethyl)-4-amino-1,2,4-triazole bromide substance and the standard sample, obtained by the state enterprise "plant of chemical reagents" of scientific and technological complex "institute of single crystals" of the National Academy of Sciences of Ukraine, was used. In the course of our work, we used chemical, physical, physicochemical (absorption spectrophotometry in the UV region), and statistical methods of analysis. The study was carried out at the Laboratory for Standardization and Technology of Medicines at the Department of Pharmaceutical Chemistry at Zaporizhzhia State Medical University (Ukraine).

Results: In the previous studies, we have developed a technique for spectrophotometric determination of the quantitative content of 1 -( $\beta$-phenylethyl)4-amino-1,2,4-triazole bromide substance. The results of the studies showed that the absorption curve in the UV region of the substance solution has three absorption maxima, namely: $\lambda_{1}=252 \mathrm{~nm}, \lambda_{2}=258 \mathrm{~nm}$, and $\lambda_{3}=263 \mathrm{~nm}$. To quantify the substance, the wavelength $\lambda_{2}=258 \mathrm{~nm}$ was used. The results of the quantitation of the 1 - $(\beta$-phenylethyl)-4-amino-1,2,4-triazole bromide substance by spectrophotometric method prove that the technique developed by us is accurate and reproducible and meets the current requirements. To the developed methodology to ensure accurate and accurate analysis results, its validation was carried out. According to the requirements of the State Pharmacopoeia of Ukraine ( 2 editions), the developed method for quantitation of the substance was checked for such validation characteristics as specificity, linearity, range of application, accuracy, correctness, and robustness.

Conclusions: As a result of the work, it was proved that the method of quantitation of 1-( $\beta$-phenylethyl)-4-amino-1,2,4-triazole bromide substance by spectrophotometric method is valid.

Keywords: Arterial hypertension, Spectrophotometry, Quantitation, Validation, 1-( $\beta$-phenylethyl)-4-amino-1,2,4-triazole bromide.

(C) 2018 The Authors. Published by Innovare Academic Sciences Pvt Ltd. This is an open access article under the CC BY license (http://creativecommons. org/licenses/by/4. 0/) DOI: http://dx.doi.org/10.22159/ajpcr.2018.v11i2.22740

\section{INTRODUCTION}

The most common pathology of the cardiovascular system is arterial hypertension $(\mathrm{AH})$, which is found in $15-20 \%$ of the adult population of the industrialized countries of the world. $\mathrm{AH}$ is recognized as a chronic non-infectious disease and is a major risk factor for coronary artery disease [1]

Every person in his life necessarily faces with cardiovascular disorders, which most often occur in the background of circulatory disorders. Unfortunately, instead of eliminating these disorders and restoring blood circulation, modern therapy is mainly aimed only at reducing the effects of these disorders. As a result, instead of cure, only inhibition of pathology is achieved and the life-long dependence of the patient on the drugs arises. Constant ingestion of chemicals due to their pronounced side effects causes more and more additional diseases [2,3].

In this regard, the creation of new highly effective medicines is an extremely important task of the pharmaceutical sciences. The foregoing prompted the creation of a fundamentally new domestic anti-anginal and antihypertensive drug, which would have a minimum of side effects. The new original compound - 1-( $\beta$-phenylethyl $)-4$-amino-1,2,4triazole bromide (conventional name "Hypertril") - was obtained at the Department of Pharmaceutical Chemistry of the Zaporizhzhia State Medical University (ZSMU) in cooperation with the SPA "Farmatron" under the direction of Professor Masur I. A. It shows combined properties of the cardioselective $\beta$ - 1 adrenergic blocking agent and peripheral vasodilator, meaning antihypertensive, anti-ischemic, and antioxidant properties $[4,5]$.

It is known that, for the development of a new drug, special attention is paid to the standardization of the active substance, which includes the study of organoleptic, physical properties, determination of the adequate quality, identification, and quantitation [6].

In our previous studies, a technique for spectrophotometric determination of the quantitative content of the substance of bromide 1 -( $\beta$-phenylethyl)-4-amino-1,2,4-triazole was developed for the $1^{\text {st }}$ time. Therefore, the purpose of our work was to determine the validation characteristics for the developed method of quantitation of 1-( $\beta$-phenylethyl)-4-amino-1,2,4-triazole bromide substance by absorption spectrophotometry in the ultraviolet (UV) region.

\section{MATERIALS AND METHODS}

For study purposes, the 1-( $\beta$-phenylethyl)-4-amino-1,2,4-triazole bromide substance and the standard sample, obtained by the state enterprise "plant of chemical reagents" of Scientific and Technological Complex "Institute of single crystals" of National Academy of Sciences of Ukraine, were used. In the course of our work, we used chemical, physical, physicochemical (absorption spectrophotometry in the UV region), and statistical methods of analysis. The study was carried out at the laboratory 
Table 1: Stability of the solution of 1-(ß-phenylethyl)-4-amino-1,2,4-triazole bromide substance in time

\begin{tabular}{llllllllll}
\hline $\mathbf{t}$ min & $\mathbf{0}$ & $\mathbf{1 5}$ & $\mathbf{3 0}$ & $\mathbf{4 5}$ & $\mathbf{6 0}$ & $\mathbf{M e a n}$ & $\mathbf{R S D t} \mathbf{\%}$ & $\mathbf{\Delta t}, \mathbf{\%}$ & $\mathbf{m a x} \boldsymbol{0} \mathbf{\%}$ \\
\hline $\mathrm{A}_{0}$ & 0.387 & 0.387 & 0.386 & 0.387 & 0.387 & 0.387 & 0.12 & 0.26 & 0.80 \\
$\mathrm{~A}$ & 0.388 & 0.389 & 0.389 & 0.389 & 0.389 & 0.389 & 0.12 & 0.26 & \\
\hline
\end{tabular}

Table 2: Metrological characteristics of linear dependence $\mathbf{y}=\mathbf{a}+\mathbf{b x}$

\begin{tabular}{|c|c|c|c|}
\hline $\begin{array}{l}\text { Value } \\
\text { name }\end{array}$ & $\begin{array}{l}\text { Data } \\
\text { value }\end{array}$ & $\begin{array}{l}\text { Criteria (for } \\
98.5-101.0 \% \text { allowance, } \\
\text { number of points 9) }\end{array}$ & $\begin{array}{l}\text { Result (match or } \\
\text { does not match) }\end{array}$ \\
\hline B & 0.9941 & - & - \\
\hline $\mathrm{s}_{\mathrm{b}}$ & 0.0091 & - & - \\
\hline $\mathrm{A}^{\mathrm{b}}$ & 0.5515 & $\leq 1.8946 * \mathrm{~S}=1.83$ & Match \\
\hline $\mathrm{s}_{\mathrm{a}}$ & 0.9643 & - & - \\
\hline $\mathrm{s}_{\mathrm{r}}$ & 0.5706 & $\leq 1.24$ & - \\
\hline $\mathrm{R}^{\mathrm{r}}$ & 0.99970 & $\geq 0.99535$ & Match \\
\hline
\end{tabular}

for standardization and technology of medicines at the Department of Pharmaceutical Chemistry at Zaporizhzhia State Medical University [6].

A particular interest, in addition to chemical methods of analysis, is caused by instrumental methods of analysis, in particular spectral. The advantages of spectral analysis methods include the objectivity, high sensitivity and accuracy of measurements, and selectivity. Furthermore, spectral methods are characterized by a small duration of analysis and the possibility of their automation and computerization, which greatly simplifies the analysis process $[6,7]$.

\section{RESULTS AND DISCUSSION}

In the previous studies, we have developed a technique for spectrophotometric determination of the quantitative content of 1-( $\beta$-phenylethyl)-4-amino-1,2,4-triazole bromide substance. The results of the studies showed that the absorption curve in the UV region of the substance solution has three absorption maxima, namely: $\lambda_{1}=252 \mathrm{~nm}, \lambda_{2}=258 \mathrm{~nm}$, and $\lambda_{3}=263 \mathrm{~nm}$. To quantify the substance, the wavelength $\lambda_{2}=258 \mathrm{~nm}$ was used [8].

The results of the quantitation of the 1-( $\beta$-phenylethyl)-4-amino-1,2,4triazole bromide substance by spectrophotometric method prove that the technique developed by us is accurate and reproducible and meets the current requirements.

To the developed methodology to ensure accurate and accurate analysis results, its validation was carried out. According to the requirements of the SPU (2 ed.), the developed method for quantitation of the substance was checked for such validation characteristics as specificity, linearity, range of application, accuracy, correctness, and robustness [6,9].

The specificity of the developed method for quantitation of 1-(ß-phenylethyl)-4-amino-1,2,4-triazole bromide substance was established by the preparation of model mixtures of the 4-amino-1,2,4triazole accompanying mixture in the maximum permissible limits and analysis of "placebo." The results of the studies have shown that the accompanying mixture almost does not absorb radiation in the spectral region, in which the absorption maxima of the studied substance are observed, and their contribution to the optical density of the samples is $<1 \%$.

Testing the stability of the test substance solution is one of the elements of studying of the robustness of the technique and should be performed before the start of all other validation studies. As a result of the study, we have proven that the test solution is stable for at least an hour. For this reason, parallel measurements of optical density of test solution and comparison solution were carried out at $\mathrm{t}=0,15,30$, 45 , and $60 \mathrm{~min}$. The results of the stability test of the solution of the 1-(ß-phenylethyl)-4-amino-1,2,4-triazole bromide substance in time are given in Table 1 .

The linear dependence was studied within the range of application of the developed method. It was confirmed directly on the substance, by preparation of standard solutions ( 9 solutions, in accordance with the requirements of the State Pharmacological Center, $2^{\text {nd }}$ edition). Based on the obtained data, we made the dependences graph of the absorbance of the obtained solutions on their concentration (Fig. 1).

The obtained results were processed using the least squares method and the linear regression equation was calculated, which had a general form: $y=a+b x$, where $y$ is the measured value (optical density), $x$ is the concentration of the determined drug, $a$ is a free line of linear dependence for the calculated regression line, and $b$ is the angular coefficient for the calculated regression line. To quantify the reproducibility of the results of the definitions and the adequacy of the linear dependence, the numerical values of the linear dependence were calculated, namely, the correlation coefficient $r$ (should be greater than the general Rc correlation index), the residual standard deviation $\mathrm{S}_{0}$ (should not exceed the maximum indeterminateness of the analysis $\Delta \mathrm{A}$ ), the free member of the linear regression $\mathrm{a}(|\mathrm{a}| \leq \Delta \mathrm{a})$, and the regression coefficient $\mathrm{b}$ (Table 2 ).

Calculated numerical values of linear dependence indicate that the linearity meets the requirements of the SPU in the selected concentration ranges.

According to the requirements of the SPU, such validation characteristics as the correctness and accuracy of the developed method of quantitation of 1-(ß-phenylethyl)-4-amino-1,2,4-triazole bromide substance by spectrophotometry method were established by the method of model mixtures (Table 3). The general objective in determining the correctness of the techniques is to identify possible systematic errors that arise as a result of influencing the results of definitions of excipients that are part of the drug.

Precision (intralaboratory accuracy) of the technique is determined by the proximity of the results for a series of measurements performed according to this method in different samples of the same homogeneous sample and is conditioned by the presence of random errors. According to the SFU, the precision of the technique is considered at three levels: Intralaboratory precision, convergence, and reproducibility. In this study, the precision of the proposed method was determined on the level of convergence. For this purpose, in each case, fifteen parallel definitions were carried out (five weighting and five repetitions), and according to the results, metrological characteristics were calculated. According to the data presented in Table 4, it was established that, in all cases, the one-sided confidence interval does not exceed the maximum permissible in determinateness of the analysis. Therefore, the methodology is exact at the level of convergence.

The range of application of the analytical technique is the interval between the minimum and maximum concentrations of the test substance, for which it is shown that the technique has the required linearity, correctness, and precision. According to the results of the conducted research, application ranges for the developed techniques are within the working intervals for the methods of quantitative determination according to the requirements of SPU (80-120\%).

The assessment of robustness was carried out at the stage of developing the methodology by identifying the factors that influence the value of optical density. The influence of these factors was taken into account when choosing the best conditions for determination $[6,10]$. 
Table 3: Results of analysis of model solutions and their statistical processing

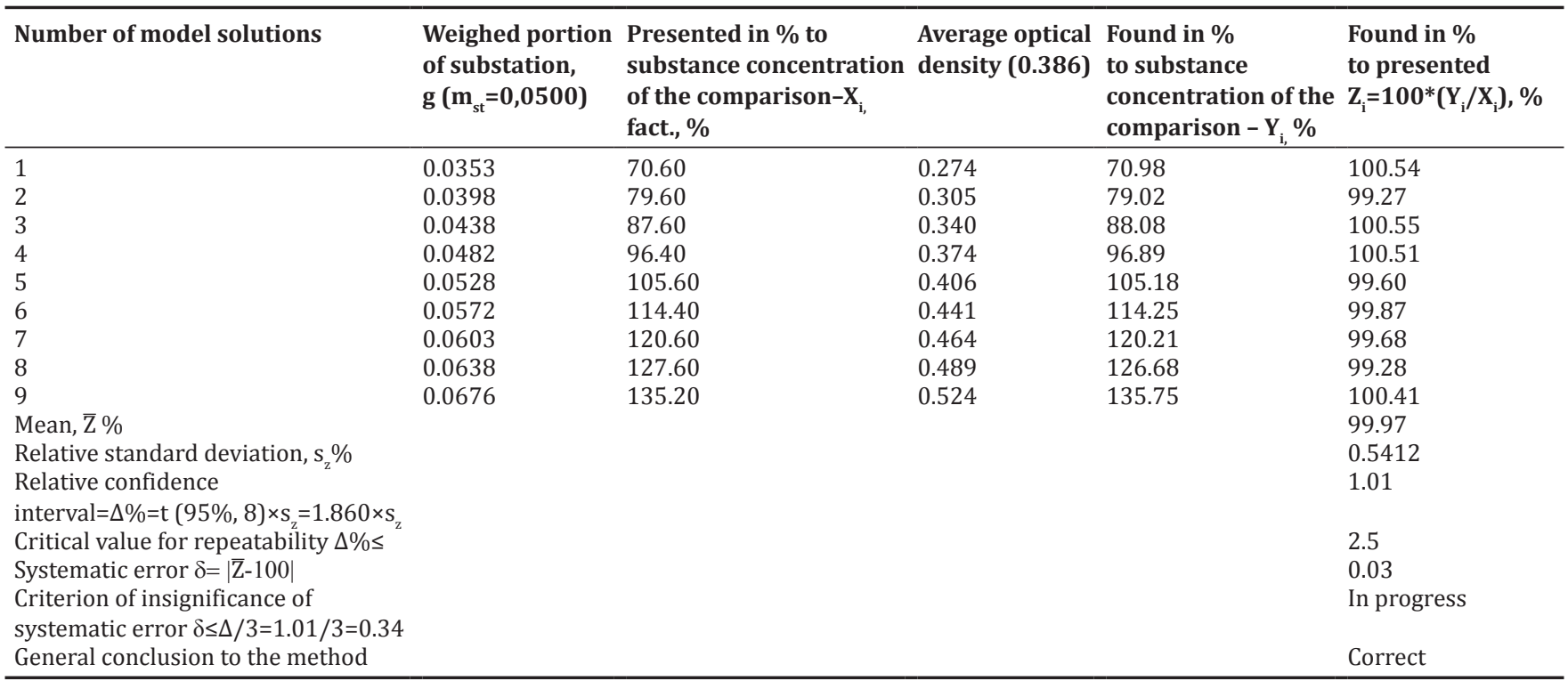

Table 4: Results of in-laboratory precision testing

\begin{tabular}{llll}
\hline Solution number & \multicolumn{2}{l}{ Values Zi } \\
\cline { 2 - 4 } & 1 study & 2 study & 3 study \\
\hline 1 & 99.58 & 100.25 & 99.25 \\
2 & 99.86 & 100.43 & 99.12 \\
3 & 99.89 & 100.12 & 99.29 \\
4 & 99.72 & 100.18 & 99.36 \\
5 & 99.42 & 100.37 & 99.31 \\
Mean & 99.69 & 100.27 & 99.27 \\
Combined mean $\mathrm{Z}_{\text {intra }} \%$ & 99.74 & & \\
$\mathrm{~S}_{\mathrm{z}}(\%)$ & 0.20 & 0.13 & 0.09 \\
$\mathrm{SD}_{\mathrm{z}}(\%)$ & 0.12 & & \\
$\Delta_{\text {intra }} \%(\mathrm{k}=5)$ & $=1.76^{*} 0.12=0.21 \leq 2.5$ & \\
\hline
\end{tabular}

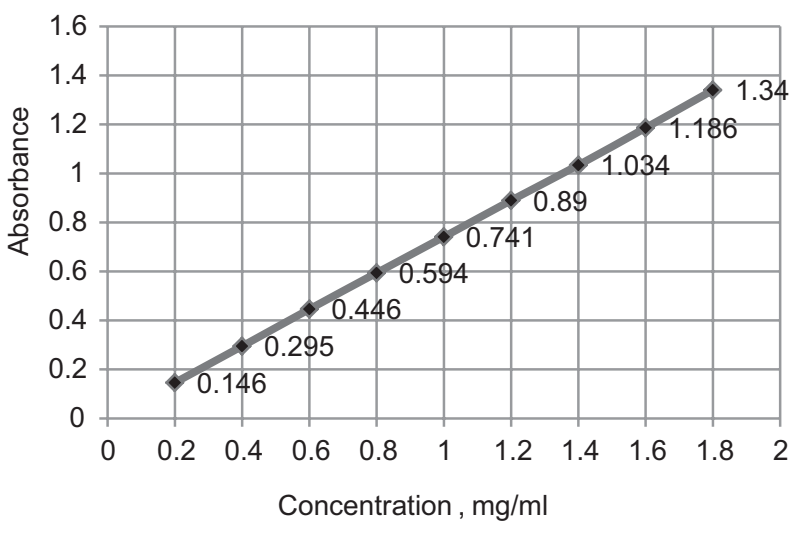

Fig. 1: Linear dependence of the absorbance on the concentration of 1-(ß-phenylethyl)-4-amino-1,2,4-triazole bromide substance

As a result of the work, it was proved that the method of quantitation of 1-( $\beta$-phenylethyl)-4-amino-1,2,4-triazole bromide substance according to characteristics such as specificity, linearity, correctness, range of application, precision, and robustness is valid.

The developed method of determination of substance by absorption spectrophotometry in the UV region will be used later for the development of methods of analysis of injectable and tableted medicinal forms of 1-( $\beta$-phenylethyl)-4-amino-1,2,4-triazole bromide.

\section{CONCLUSION}

In the course of the work, the data of the scientific literature on the distribution of the incidence of the population of Ukraine $\mathrm{AH}$ and ischemic heart disease and the prospects for the creation of new antihypertensive and antiangial drugs are analyzed. Furthermore, the method of quantitation of 1-( $\beta$-phenylethyl)-4-amino-1,2,4-triazole bromide substance by spectrophotometric method according to the requirements of SFU ( $2^{\text {nd }} \mathrm{ed}$.) was validated. The results of the conducted work proved that the developed method for such characteristics as specificity, linearity, correctness, range of application, precision, and robustness is valid.

\section{AUTHORS CONTRIBUTION}

The article is a product of the intellectual environment of the whole team; and that all members have contributed in various degrees to the analytical methods used, to the research concept, and to the experiment design.

\section{CONFLICTS OF INTERESTS}

All authors have none to declare.

\section{REFERENCES}

1. Blood Pressure Lowering Treatment Trialists' Collaboration. Blood pressure-lowering treatment based on cardiovascular risk: A metaanalysis of individual patient data. Lancet 2014;384:591-8.

2. White W. Blood Pressure Monitoring in Cardiovascular Medicine and Therapeutscs. N. Jersey: Humana Press; 2001.

3. Mazur I, Chekman I, Belenichev I. Metabolithotropic Drugs. Ukraine: Zaporozhye; 2007.

4. Belenichev I, Kucherenko L, Nagorna O, Yu V, Mazur I, Parnyuk N. Metabolite tropic mechanisms of cardioprotective effect of new antianginal and antihypertensive drug "Hypertril" in experimental ischemia of myocardium. Odessa Med J 2014;6(146):22-6.

5. Belenichev I, Volchek YU, Kucherenko L, Nagornaya E, Parnyuk N. Research activity ß-adrenoceptor blocking in the series of 1-alkyl (carboxyalkyl)-4-ylideneamino-1,2,4-triazole. Exp Clin Physiol Biochem 2014;3:24-32.

6. Ukrainian Scientific Pharmacopoeial Center for Quality of Medicines. State Pharmacopoeia of Ukraine. $2^{\text {nd }}$ ed., Vol. 1. Kharkiv: Ukrainian Scientific Pharmacopoeial Center for Quality of Medicines, Ukrainian; 
2015. p. 85-100.

7. Beckett AH, Stenlake JB. UV-Visible Spectrophotometry: Practical Pharmaceutical Chemistry. $4^{\text {th }}$ ed., Vol. 2. New Delhi: C.B.S. Publishers; 2001. p. 285-97.

8. Kucherenko L. On the standardization of hypertril substance. Pharm Rev 2015;2:64-7.

9. Manjunath S, Chouhan V, Sandeep S. Spectrophotometric estimation of levosulpiride in bulk drug and formulations. Int J Pharm Pharm Sci 2011;3:135-7

10. Nayak SC, Kulkarni PV, Bhaskar V, Chavhan V. Development and validation of UV spectrophotometric method for simultaneous estimation of doxylamine succinate and pyridoxine hydrochloride in bulk and tablet dosage form. Int J Pharm Pharm Sci 2013;5:390-3. 